\title{
Journal of Clinical Case Reports
}

\section{Primary Skin Umbilical Endometriosis: About One Case David Boccara*, Antoine De Runz, Oren Marco, Marc Chaouat and Maurice Mimoun \\ Hospital Saint-Louis, Paris, France}

\begin{abstract}
Extremely rare ( $1 \%$ of the cases), cutaneous endometriosis can be located in the umbilical region. Its principal differential diagnoses are keloid scar, sebaceous cyst and melanoma which must first be discounted. Most often in a secondary location and accompanied by concomitant menstrual pain and bleeding following abdominal surgery, Villar's nodule can also be totally asymptomatic, isolated and without a known cause. This was the distinctive feature of our 33-year-old patient whose nodule had appeared 5 years beforehand. Only histological examination could confirm a diagnosis of endometriosis - even if the bluish multinodular aspect had strongly suggested it. Extended excision is the treatment of choice.
\end{abstract}

Keywords: Endometriosis; Primary; Skin; Umbilical; Villar

\section{Introduction}

Endometriosis is a frequent pathology that affects over 10\% of menstruating women. It is characterized by the presence of endometrial tissue outside of the uterine cavity. While a few distant locations have been described such as the lungs and the brain, endometriosis most often affects the ovaries, uterosacral ligaments, rectum and the bladder. The causes of endometriosis are poorly understood. However, retrograde menstruation is the principle hypothesis. The blood circulating during menstruation flows back through the fallopian tubes and into the abdominal cavity. This phenomenon does not explain everything since $90 \%$ of women have retrograde bleeding whereas only $10 \%$ have endometriosis. Studies are currently being performed to determine a possible genetic predisposition [1]. Cutaneous, subcutaneous and muscular locations are rare. A few cases have been described in the literature [2,3]. Also called Villar's nodule, umbilical endometriosis was described for the first time in 1886. It represents less than $1 \%$ of extragenital cases. Subject to hormonal variations [4] like the rest of uterine tissue, it can be totally asymptomatic [5-7]. However, it is most often diagnosed following chronic pelvic pain or within the framework of an infertility work-up [8].

With cutaneous and subcutaneous lesions, pain can be recurrent and sometimes very acute during menstruation, accompanied or not by bleeding. Clinically, the lesions are most often nodular, multiple, bluish and hard, on or around an old scar following pelvic surgery, a cesarean, laparoscopy or even amniocentesis [9-12]. Treatment consists of surgical excision.

\section{Case report}

A 33-year-old patient, nulliparous and with no past history, was referred to us by her gynecologist for suspicion of sebaceous cyst which had appeared 5 years beforehand, frequently accompanied by pain and swelling, and recently confirmed by ultrasound. With each bout, symptoms lasted approximately 8 days without concurrence with menstruations. Following a recent increase in the size of the lesion, an imaging work-up was performed. Neither endovaginal and abdominal ultrasound nor abdominopelvic CT revealed endometrial lesions. Given the absence of both an endometrial lesion on CT and ultrasound and previous abdominopelvic surgery, a diagnosis of endometriosis was initially discounted by her doctor. Moreover, the patient had never miscarried or presented dyspareunia. Owing to the aspect of these bluish nodules measuring $1 \mathrm{~cm}, 2 \mathrm{~cm}$ and $3 \mathrm{~cm}$, we decided to perform surgical excision under local anesthetic. Owing to the very suggestive similarity to Villar's nodule, excision was performed with $5-\mathrm{mm}$ margins and all fat and muscle up to the peritoneum were removed.
Clinically, the nodule only involved the skin and subcutaneous fat. It was not in contact with the underlying muscular aponeurosis. Initially closure was performed by simple cutaneous plasty by drawing in the borders with the navel reconstructed by simple cutaneous plasty. The nodule was sent for anatomopathological examination which confirmed the diagnosis of umbilical endometriosis. A subsequent physical examination then found no other cutaneous or subcutaneous endometrial nodule. Secondly, we have done a new umbilicus with a tennis ball flap. On follow-up six months later, healing was complete and the patient was satisfied with the aesthetic result (Figures 1-7).

\section{Discussion}

Villar's nodule is rarely the first diagnosis evoked. In our patient,

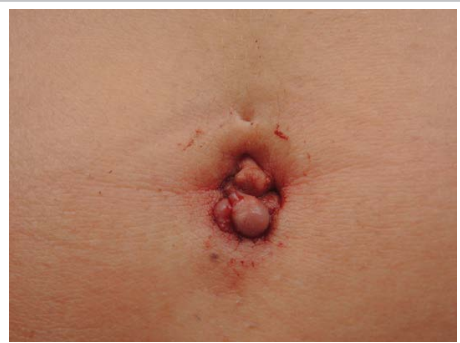

Figure 1: Umbilical endometriosis nodule.

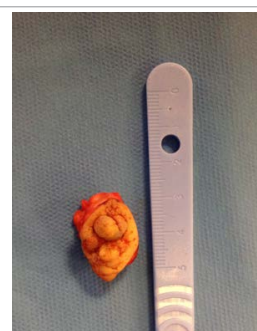

Figure 2: Excision of the nodule.

*Corresponding author: David Boccara, Hospital Saint-Louis, Paris, France, Tel: +33 (0)142385044; E-mail: davboc9@hotmail.com

Received February 04, 2016; Accepted March 23, 2016; Published March 28 2016

Citation: Boccara D, Runz AD, Marco O, Chaouat M, Mimoun M (2016) Primary Skin Umbilical Endometriosis: About One Case. J Clin Case Rep 6: 742. doi:10.4172/2165-7920.1000742

Copyright: () 2016 Boccara D, et al. This is an open-access article distributed under the terms of the Creative Commons Attribution License, which permits unrestricted use, distribution, and reproduction in any medium, provided the original author and source are credited. 


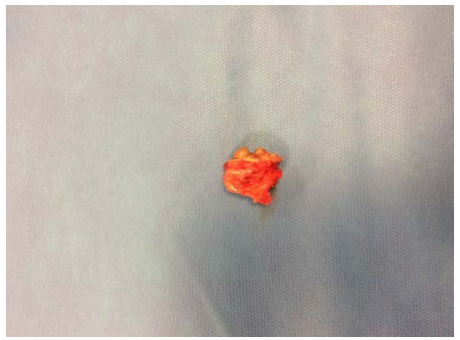

Figure 3: Excision was perfomed to the aponeurosis

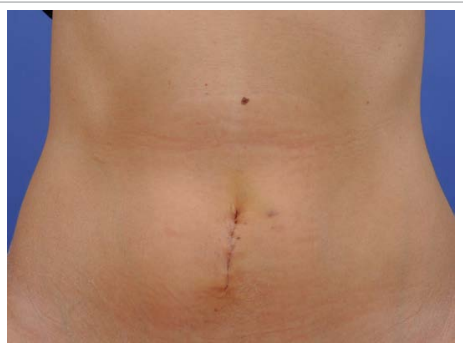

Figure 4: Initially simple closure.

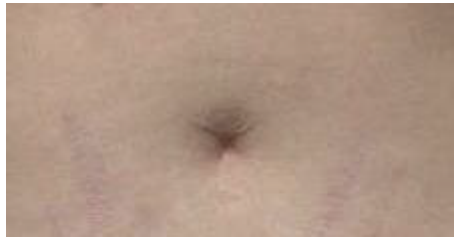

Figure 5: New Umbilicus.

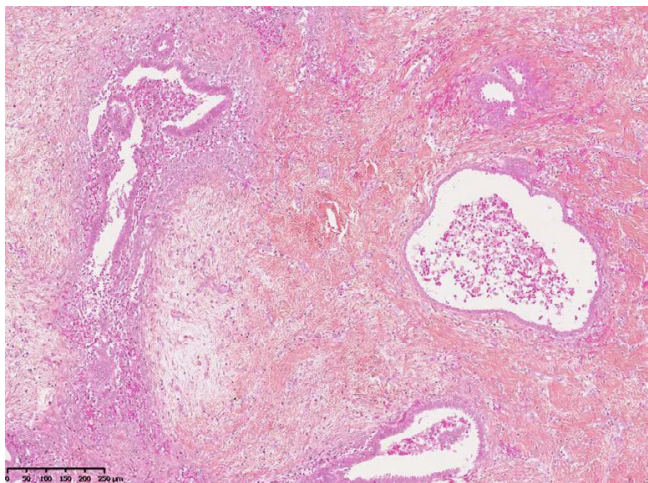

Figure 6: Endometriosis. (HE staining, x10 magnification).

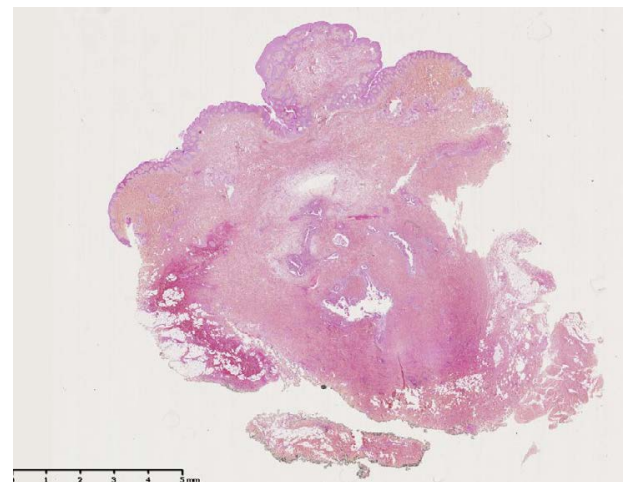

Figure 7: Endometriosis. Gland and cytogeneses chorion (HE staining, x100 magnification). there were no signs such as previous pelvic surgery, concomitant menstrual symptoms or secondary endometrial locations. This is the distinctive characteristic of our case. The two main differential diagnoses, sebaceous cyst and melanoma, were improbable in our patient. For melanoma, the age of the lesion and its lack of evolution were not in favor of an invasive malignancy. In 2007, following a review of the literature, Victory et al. concluded that the mean age that umbilical endometriosis is diagnosed is 37.7 years. The bluish nodular aspect and the age of our patient initially suggested this diagnosis. A biopsy associated with a histological examination should have probably been performed earlier. Treatment of endometriosis has not been standardized. Some authors first prescribe medical treatment such as progesterone, danazol or GnRH analogues. While these treatments do sometimes relieve symptoms such as pain and bleeding, they do not eliminate the endometriosis, in particular, its cutaneous forms. It is therefore preferable to perform surgical excision with margins that are at least $5 \mathrm{~mm}$ in diameter and depth. According to Victory et al. [8], $70 \%$ of the patients are treated in this manner.

Once the histological results are obtained, it is sometimes necessary to re-operate if the excised rims are not healthy. As for the navel, it is rare to be able to preserve it given the size of the excision that is required. Direct sutures on several levels are therefore performed. If reconstruction is necessary for aesthetic reasons, it will be performed later after the histological results have been obtained [8]. Surgery therefore provides a diagnosis and discounts primary or secondary malignancies. When the surgical procedure is complete, it makes it possible to avoid malignant transformation of endometriosis which is rare but has been described in the literature [13].

\section{Conclusion}

Umbilical endometrial nodules only represent $1 \%$ of extra genital lesions and are rarely initially diagnosed. They are easily diagnosed when there is a history of abdominal surgery and associated menstrual symptoms such as pain and bleeding. However, certain cutaneous lesions can be isolated with no evocative clinical signs or previous pelvic surgery. While a pelvic location is the most frequent, an umbilical location can be isolated as was the case for our patient. In that case, diagnosis is often difficult. In case of fortuitous diagnosis, it is essential to perform a complete gynecological work-up to make sure that the patient does not present a gynecological location that could favor infertility.

\section{References}

1. Gajjar KB, Mahendru AA, Khaled MA (2008) Caesarean scar endometriosis presenting as an acute abdomen: a case report and review of literature. Arch Gynecol Obstet 277:167-169.

2. Coley BD, Casola G (1993) Incisional endometrioma involving the rectus abdominis muscle and subcutaneous tissues: CT appearance. AJR Am J Roentgenol 160:549-550.

3. Fancellu A, Pinna A, Manca A, Capobianco G, Porcu A (2013) Primary umbilical endometriosis. Case report and discussion management options. Int J Surg Case Rep 4: 1145-1148.

4. Králičková M, Vetvicka V (2014) Endometriosis and ovarian cancer.World J Clin Oncol 5:800.

5. Khaled A, Hammami H, Fazaa B, Zermani R, Ben Jilani S, et al. (2008) Primary umbilical endometriosis: a rare variant of extragenital endometriosis. Pathologica 100: 473-475.

6. Elabsi M, Lahlou MK, Rouas L (2002) L'endométriose cicatricielle de la paroi abdominale. Ann Chir 127: 65-67.

7. Simsir A, Thorner K, Waisman J, Cangiarella J (2001) Endometriosis in 
Citation: Boccara D, Runz AD, Marco O, Chaouat M, Mimoun M (2016) Primary Skin Umbilical Endometriosis: About One Case. J Clin Case Rep 6: 742. doi:10.4172/2165-7920.1000742

abdominal scars: a report of three cases diagnosed by fine-needle aspiration biopsy. Am Surg 67: 984-986.

8. Victory R, Diamond MP, Johns DA (2007) Villar's nodule: a case report and systematic literature review of endometriosis externa of the umbilicus. J Minim Invasive Gynecol 14: 23-32.

9. Thylan S (1995) Cutaneous and subcutaneous endometriosis. Dermatol Surg $21: 737$.

10. Purvis RS, Tyring SK (1994) Cutaneous and subcutaneous endometriosis Surgical and hormonal therapy. J Dermatol Surg Oncol 20: 693-695.
11. Victory R, Diamond MP, Johns DA (2007) Villar's nodule: a case report and systematic literature review of endometriosis externa of the umbilicus. J Minim Invasive Gynecol 14: 23-32.

12. Gajjar KB, Mahendru AA, Khaled MA (2008) Caesarean scar endometriosis presenting as an acute abdomen: a case report and review of literature. Arch Gynecol Obstet 277: 167-169.

13. Dobrosz Z, Paleń P, Stojko R, Właszczuk P, Niesłuchowska-Hoxha A, et al (2014) Clear cell carcinoma derived from an endometriosis focus in a scar after a caesarean section: a case report and literature review. Ginekol Pol 85: 792-795. 